for older service users with HIV compared with the general older population, including earlier detection of general health problems. However, services in low HIV prevalence areas were commonly considered to be youth-orientated. The targeting of sexual health/HIV resources towards younger people was identified as a key contributor to the high proportion of older adults diagnosed at a late stage of disease. Late HIV diagnosis was associated with a lack of awareness or acknowledgement of personal risk.

Discussion/conclusion Once diagnosed, older adults report receiving exceptional HIV care and support. However, to promote early HIV detection, HIV awareness needs to increase across all age groups.

\section{P136 IS 7 DAYS OF DOXYCYCLINE ENOUGH FOR THE TREATMENT OF LGV?}

Rebecca Simons*, John White. Guys and St Thomas' NHS Trust, London, UK

\section{$10.1136 /$ sextrans-2016-052718.190}

Background Current treatment recommendations for lymphogranuloma venereum (LGV) are for 21 days of doxycycline but the evidence is limited.

Aims To describe clinical outcomes in MSM treated with shortcourse doxycycline for rectal Chlamydia, subsequently proven to be LGV.

Methods We reviewed all MSM who received 7-14 days of doxycycline but tested positive for LGV between Dec 2012 - Jan 2016. Clinical features and test of cure (TOC) results were tabulated.

Results Thirty-three MSM with LGV were included. Median age was 35 (range 25-57), 88\% were HIV positive and all tested positive for LGV-specific DNA in the rectum. Only 18/33 (55\%) had ano-rectal symptoms including pain, bleeding, tenesmus, discharge, constipation, diarrhoea and ulceration. The remainder were asymptomatic or had genital symptoms. 24/33 (73\%) were treated with 7 days of doxycycline, 8 (25\%) with 14 days, and 1 patient received azithromycin $1 \mathrm{~g}$ stat; 20 patients also received ceftriaxone 500mg. 100\% (32/32) of patients treated with doxycycline had a negative TOC at a median 28 days (range 14200). Of these, 1 patient had an initial positive rectal chlamydia TOC but this was LGV negative. The patient treated with azithromycin had a positive TOC at 24 days and remained symptomatic, representing treatment failure.

Discussion/conclusion This case series suggests efficacy for shortcourse doxycycline for both symptomatic and asymptomatic rectal LGV with no treatment failures seen. Current BASHH Chlamydia guidelines suggest 21 days of doxycycline should be considered if no LGV test is done. Our data suggest this is not warranted but further prospective studies are needed.

\section{P137 IS THE MANAGEMENT OF HIV POSITIVE LYMPHOMA PATIENTS WITHIN SMALL COHORT CENTRES IN LINE WITH CURRENT BEST PRACTICE GUIDELINES?}

${ }^{1}$ Mickaela Poree*, ${ }^{3}$ Seema Malik, ${ }^{2}$ Emily Clarke, ${ }^{2}$ Rajul Patel, ${ }^{2}$ Selvavelu Samraj. ${ }^{1}$ University of Southampton, Southampton, UK; ${ }^{2}$ Solent NHS Trust, Southampton, UK; ${ }^{3}$ Bournemouth and Christchurch NHS Trust, Bournemouth, UK

10.1136/sextrans-2016-052718.191
Background As the incidence of opportunistic infections accounting for HIV-related deaths has declined significantly, malignancies now account for approximately a third of HIV related death with the majority of these being lymphoma related.

Aims To determine whether management of HIV positive patients within small centres is in line with current best practice guidelines.

Methods All HIV positive patients diagnosed with lymphoma or relapsing with lymphoma, from 2010 to 2015, in the BASHH Wessex region were eligible for inclusion. On review of previous studies and current BHIVA guidelines, a data collection tool was designed to accumulate relevant patient information to review care. Data collection was conducted at three centres across Wessex.

Results The total sample size of the study was 25 patients, all of which received the recommended diagnostic and staging procedures. Of the patients with documented staging, 73\% were diagnosed with stage 4 lymphoma and $50 \%$ had an International Prognostic Index score of 3 or more. The mean follow up time of each patient was 19 months (from 1 to 64 months). Within the follow up time, the overall survival rate was $72 \%$. In those followed up for a minimum of 2 years, the progression free survival rate was $100 \%$.

Conclusions Although the small sample size does not allow comparison with larger cohorts, current findings suggest that management of HIV positive lymphoma patients within these smaller centres is in line with best care guidelines. The formation of a national registry would allow for closer monitoring of this in the future.

\section{\begin{tabular}{|l|l} 
P138 IDENTIFYING THE PREVALENCE OF RECREATIONAL \\
\hline
\end{tabular} DRUG USE IN MEN WHO HAVE SEX WITH MEN ATTENDING SEXUAL HEALTH CLINICS IN GREATER MANCHESTER}

${ }^{1}$ Andrew Tomkins*, ${ }^{2}$ Sameena Ahmad, ${ }^{2}$ Luke Cannon, ${ }^{3}$ Stephen P Higgins, ${ }^{1}$ Merav Kilner,
${ }^{3}$ Anastasia Kolyva, ${ }^{4}$ Chris Ward, ${ }^{1}$ Roberto Vivancos. ${ }^{1}$ Public Health England-North West;
${ }^{2}$ University Hospital South Mancheste; ${ }^{3}$ Pennine Acute Hospitals Trust; ${ }^{4}$ Central Manchester ${ }^{2}$ University Hospital
Foundation Trust

10.1136/sextrans-2016-052718.192

Background/introduction Recreational drug use has been reported to be disproportionately higher in men who have sex with men (MSM) when compared to their heterosexual counterparts. There are indications that increased recreational drug use may lead to risky sexual behaviour. Patterns of recreational drug use are subject to spatial and temporal variations and as such, regular assessment of these changes is essential in understanding service user behaviour.

Aim(s)/objectives To identify commonly used recreational drugs, routes of administration and frequency of use for MSM service users attending sexual health clinics in Greater Manchester.

Methods A retrospective case note review was conducted using a random, powered sample of MSM attending three sexual health clinics across Greater Manchester during 2014. Details of risky sexual practices, sexually transmitted infection diagnoses and number of sexual partners were also recorded.

Results 357 case notes were reviewed from across three sites. In total, $18 \%$ of service users reported recreational drug use. The most commonly reported substances were cannabis (7\%) and 
cocaine (6\%). $3.7 \%$ of service users reported using at least one of the three main drugs typically associated with chemsex.

Discussion/conclusion Our study identified that substance misuse is common in MSM attending sexual health clinics in Greater Manchester. It highlights the need for the robust collection of data during consultation in order to better understand service user requirements.

\section{P139 SCREENING FOR CHILD EXPLOITATION IN ONLINE SEXUAL HEALTH SERVICES: AN EXPLORATORY STUDY OF EXPERT VIEWS}

${ }^{2}$ Victoria Spencer-Hughes, ${ }^{1} J$ onathan Syred, ${ }^{2}$ Gillian Holdsworth, ${ }^{1}$ Paula Baraitser*. ${ }^{*}$ Kings College London, London, UK; ${ }^{2}$ Lambeth and Southwark Public Health Directorate, London, UK

\subsection{6/sextrans-2016-052718.193}

Background/introduction Sexual health services routinely screen for child sexual exploitation. Although, sexual health services are increasingly provided online there has been no research on translation of the safeguarding function to online services. We studied expert views on safeguarding in this context.

Aim(s)/objectives To explore expert views on safeguarding within online sexual health services and their implications for service development.

Methods We conducted semi-structured interviews with local, regional and national experts purposively sampled to represent a wide range of organisations that have direct influence over CSE protocols, child protection policies and sexual health services. Interviews were analysed by three researchers using a matrix based analytic method.

Results Our respondents described two different approaches to safeguarding. The 'information providing' approach considers that young people, at risk of CSE, will ask for help, when they are ready from someone they trust. The primary function of the service is to provide information, generate trust and respond reliably to disclosure. The approach values online services as an anonymous space to test out disclosure without commitment. The 'information gathering' approach considers that young people may withhold information about exploitation. Services should therefore seek out information to assess risk and initiate disclosure. This approach values face-to-face opportunities for individualised questioning and immediate referral.

Discussion/conclusion The 'information providing' approach is associated with confidential telephone support lines and the 'information gathering' approach with clinical services. The approach adopted online will depend on ethos and the range of services provided. Effective transition from online to clinic services after disclosure is an essential element of this process and further research is needed to understand and support this transition.

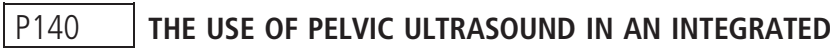 SEXUAL HEALTH SERVICE}

Rachael Viney*, Mayur Chauhan. Newcastle Hospitals NHS Foundation Trust, Newcastle, UK

\subsection{6/sextrans-2016-052718.194}

Background Clients presenting to integrated sexual health services may have gynaecological and contraceptive problems requiring ultrasound assessment. This would usually need referral to radiology causing delays in diagnosis and engendering patient worry and anxiety. To address this we have developed an inhouse ultrasound service.

Aim To analyse if the use of pelvic ultrasound improves the patient journey, avoids referrals to radiology and saves time and potentially money.

Methods Over a 6-month period, 180 transvaginal pelvic ultrasounds were performed. So far we have reviewed 50 case notes. Information collated includes the indication for the scan, the findings and diagnosis. Further analysis of the rest is on going.

Results Preliminary results show that $96 \%$ of patients had their ultrasound on the day of initial presentation. Some of the indications for scanning included pelvic pain (36\%), confirmation of position of IUC (30\%) and abnormal bleeding (10\%). $88 \%$ of patients were managed within the sexual health service and did not require onward referral. The majority of these had normal scans. Abnormal findings on scanning included fibroids, partial uterine perforation, adenomysosis and polycystic ovaries. 6 patients required referrals; one for a urological problem and 5 for appropriate gynaecological problems such as endometriosis and pelvic congestion syndrome. No radiology departmental ultrasound scans were required.

Conclusion Use of ultrasound in an integrated sexual health service provides patients with a streamlined experience, effectively providing a 'one stop shop' for most sexual health presentations. In the long run it could provide a value-based local service.

\section{P141 A SERVICE EVALUATION FOR AND ON BEHALF OF THE EUROPEAN CLINICAL COLLABORATIVE GROUP (ECCG) - THE MANAGEMENT OF GONORRHOEA ACROSS EUROPE}

\begin{abstract}
${ }^{1}$ Hibak Aadan*, 'James Mawdsley, ${ }^{1}$ Harriet Eatwell, ${ }^{2}$ Emily Clarke, ${ }^{3}$ Mikhail Gomberg, ${ }^{4}$ Vladimir Kovalyk, ${ }^{5}$ Harold Moi, ${ }^{6}$ Airi Poder, ${ }^{7}$ Jonathan Ross, ${ }^{8}$ Magnus Unemo, ${ }^{9}$ John White, ${ }^{1,10}$ Rajul Patel. ${ }^{1}$ University of Southampton, Southampton, UK; ${ }^{2}$ Solent NHS Trust, Southampton, UK; ${ }^{3}$ Moscow State University for Medicine and Dentistry, Moscow, Russia; ${ }^{4}$ Clinical Hospital of Federal Medical and Biological Agency, Russia, Russia; ${ }^{5}$ Oslo University Hospital, University of Oslo, Norway; ${ }^{6}$ Tartu University Clinics Foundation, Clinic of Dermatovenerology, Estonia; ${ }^{7}$ University Hospital Birmingham, Birmingham, UK; ${ }^{8}$ Örebro University Hospital, Orebro, Sweden; ${ }^{9} G u y ' s$ and St Thomas' NHS Foundation Trust, London, UK; ${ }^{10}$ Royal South Hants Hospital, Southampton, UK
\end{abstract}

\subsection{6/sextrans-2016-052718.195}

Background/introduction Gonorrhoea (GC) cases appear to be rising as well as increasing problems with resistance to many antibiotic groups. With open boundaries and free movement of populations and doctors, a consistent standard of care is important across Europe. Regular evaluation is crucial in controlling the emergent spread of resistant GC.

Aim(s)/objectives To evaluate current clinical practice amongst sexual health physicians across Europe against the current European guidelines. Also, key areas of controversy will be explored with to help inform further guideline development.

Methods The ECCG is a network of 120 sexual health specialists across 38 countries, who conduct questionnaire-based research across the European region. An expert panel consisting of six ECCG members was established then interviewed to help identify areas of controversy. Subsequently, a clinical scenario based questionnaire was developed then disseminated to all ECCG members.

Results Provisional results demonstrate variation in clinical practice across Europe. This is discernable from the choice of treatment for a patient with a history of anaphylaxis to penicillin and 